\title{
A fully automated centrifugal microfluidic system for sample-to-answer viral nucleic acid testing
}

\author{
Fei $\operatorname{Tian}^{1,2 \dagger}$, Chao Liu ${ }^{1,2 \dagger}$, Jinqi Deng ${ }^{1,2}$, Ziwei Han $^{1,2}$, Lu Zhang ${ }^{3 *}$, \\ Qinghua Chen ${ }^{1 *} \&$ Jiashu Sun ${ }^{1,2^{*}}$ \\ ${ }^{1}$ CAS Key Laboratory of Standardization and Measurement of Nanotechnology, CAS Center for Excellence in Nanoscience, \\ National Center for Nanoscience and Technology, Beijing 100190, China; \\ ${ }^{2}$ University of Chinese Academy of Sciences, Beijing 100049, China; \\ ${ }^{3}$ Department of Chemistry, Capital Normal University, Beijing 100048, China
}

Received May 7, 2020; accepted June 23, 2020; published online July 27, 2020

\begin{abstract}
The outbreak of virus-induced infectious diseases poses a global public-health challenge. Nucleic acid amplification testing (NAAT) enables early detection of pandemic viruses and plays a vital role in preventing onward transmission. However, the requirement of skilled operators, expensive instrumentation, and biosafety laboratories has hindered the use of NAAT for screening and diagnosis of suspected patients. Here we report development of a fully automated centrifugal microfluidic system with sample-in-answer-out capability for sensitive, specific, and rapid viral nucleic acid testing. The release of nucleic acids and the subsequent reverse transcription loop-mediated isothermal amplification (RT-LAMP) were integrated into the reaction units of a microfluidic disc. The whole processing steps such as injection of reagents, fluid actuation by rotation, heating and temperature control, and detection of fluorescence signals were carried out automatically by a customized instrument. We validate the centrifugal microfluidic system using oropharyngeal swab samples spiked with severe acute respiratory syndrome coronavirus 2 (SARS-CoV-2) armored RNA particles. The estimated limit of detection for armored RNA particles is 2 copies per reaction, the throughput is 21 reactions per disc, and the assay sample-to-answer time is approximately 70 min. This enclosed and automated microfluidic system efficiently avoids viral contamination of aerosol, and can be readily adapted for virus detection outside the diagnostic laboratory.
\end{abstract}

nucleic acid testing, virus, microfluidics, sample-to-answer, automation

Citation: Tian F, Liu C, Deng J, Han Z, Zhang L, Chen Q, Sun J. A fully automated centrifugal microfluidic system for sample-to-answer viral nucleic acid testing. Sci China Chem, 2020, 63: 1498-1506, https://doi.org/10.1007/s11426-020-9800-6

\section{Introduction}

Infectious diseases caused by pathogens are one of the leading causes of death in the world. According to the statistics provided by the world health organization (WHO), the number of deaths attributable to pathogen infections such as hepatitis, tuberculosis and malaria is 4.3 million worldwide

$†$ These authors contributed equally to this work.

*Corresponding authors (email: zhangl@cnu.edu.cn; chenqh@nanoctr.cn; sunjs@nanoctr.cn) in 2016 [1]. The emergence and spread of pathogens such as Middle East respiratory syndrome (MERS) coronavirus, Ebola virus, H1N1 virus, Zika virus, and severe acute respiratory syndrome coronavirus 2 (SARS-CoV-2) severely affect global public health. Early detection of these pathogens is of great importance in providing timely treatment and preventing onward transmission [2-4]. Especially in a virusinduced public health emergency, there is an urgent need to develop accurate, rapid, and on-site assays for screening suspected patients and diagnosing viral infection [5]. 
Nucleic acid amplification testing (NAAT) is an effective tool for clinical pathogen detection, owing to its capability of specific amplification and sensitive identification of target sequences [6-10]. Compared with polymerase chain reaction in detecting pathogens [11], isothermal nucleic acid amplification obviates the need for thermocycling [12-14], making NAAT more rapid and convenient [15-17]. However, the general requirements for repetitive manual operations such as nucleic acid extraction and amplification, expensive instrumentation, and high-level biosafety laboratories have hindered the use of NAAT for screening and diagnosis of infectious diseases outside the clinical diagnostic laboratory [5].

Microfluidic technology capable of manipulating small volumes of fluid and integrating a variety of reactions holds great promise for viral nucleic acid testing [18-29]. For example, microfluidics combined with a commercially available coffee mug or portable heating device allowed for sensitive and rapid detection of Zika virus by integrating RNA extraction, enrichment, and amplification in a microfluidic device [30-32]. In another work, a multifunctional microfluidic device pre-loaded with wax-sealed reagents was designed for extraction and amplification of viral nucleic acids in a point-of-care format [33]. Our group presented a microcapillary-based assay for sample-to-answer detection of HIV virus [34,35]. To enable nucleic acid testing without the need of electricity, we developed a microfluidic disc combined with a hand-powered centrifugal device for sample-to-answer diagnostics of pathogens [36]. Despite the great potential of microfluidic NAAT, there is still a lack of fully automated and enclosed microfluidic systems with sample-in-answer-out capability for sensitive, specific, and rapid viral nucleic acid testing [37,38].

In this work, we develop an automated centrifugal microfluidic system (Figure 1) consisting of a microfluidic disc and a customized instrument for rapid sample-to-answer detection of SARS-CoV-2 armored RNA particles with high sensitivity and specificity. The microfluidic disc contains 21 identical and independent reaction units, each of which integrates the on-chip release of nucleic acids and reverse transcription loop-mediated isothermal amplification (RTLAMP). After injection of oropharyngeal swab samples into the microfluidic disc, the whole processing steps including sample treatment, RT-LAMP, and fluorescence signal detection were carried out automatically. This enclosed and automated microfluidic system could rapidly detect viral nucleic acid without resorting to skilled operators and diagnostic laboratories, promoting diagnosis efficiency of infectious diseases.

\section{Materials and method}

\section{$2.1 \quad$ Reagents}

The virus lysis/nucleic acid release kit was purchased from Sansure Biotech (China). The nucleic release reagent comprised of Tris-HCl, Triton X-100, Tween-20, and octylphenoxy-poly(ethoxyethanol), which can stabilize $\mathrm{pH}$ of reaction solution to a LAMP-compatible level, protect single-stranded RNA after virus lysis, and preserve the enzyme activity. Three RT-LAMP primer sets targeted to N, E, and ORF1ab (O) genes of SARS-CoV-2 and two types of plasmids containing the $\mathrm{N}$ genes of SARS-CoV or SARSCoV-2 were synthesized by Sangon Biotech (China). Bst DNA polymerase, avian myeloblastosis virus (AMV) reverse transcriptase, calcein kit, and $2 \times$ reaction buffer for RTLAMP (composed of Tris- $\mathrm{HCl}, \mathrm{pH} 8.8,40 \mathrm{mM}$; $\mathrm{KCl}$, $20 \mathrm{mM}$; $\mathrm{MgSO}_{4}, 16 \mathrm{mM}$; $\left(\mathrm{NH}_{4}\right)_{2} \mathrm{SO}_{4}, 20 \mathrm{mM}$; Tween 20, $0.2 \%$; betaine, $1.6 \mathrm{M}$; dNTPs, $2.8 \mathrm{mM}$ for each type), positive control RNA (PC RNA), and Primer Mix. RNA (PM RNA) were purchased from Eiken China (China). Tris buffer containing $1 \mathrm{M}$ Tris- $\mathrm{HCl}(\mathrm{pH}$ 8.0) was purchased from Solarbio (China). DNase-free water was purchased from Sangon Biotech (China). The mineral oil was purchased from Sigma-Aldrich (USA).
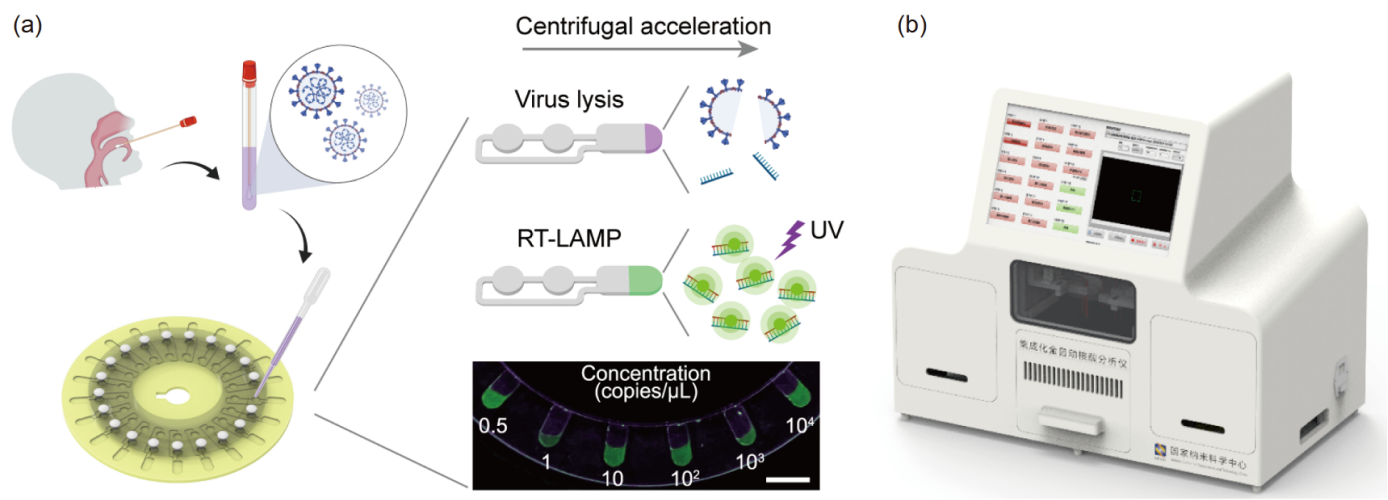

Figure 1 Overview of the automated centrifugal microfluidic system for sample-to-answer viral nucleic acid testing. (a) Procedures of viral nucleic acid testing by integrating the release of nucleic acids and reverse transcription loop-mediated isothermal amplification (RT-LAMP) detection within a microfluidic disc. Scale bar, $1 \mathrm{~cm}$. (b) Schematic of the customized instrument for automated sample-to-answer detection (color online). 


\subsection{Armored RNA construction}

The armored RNA particles are a complex of a singlestranded RNA and MS2 bacteriophage coat protein. Three types of SARS-CoV-2 armored RNA particles ( $10^{9}$ copies/ $\mathrm{mL}$ ) containing MS2 bacteriophage coat protein and 350nucleotide RNA sequence (the part sequence of $\mathrm{N}$ gene, the whole sequence of $\mathrm{E}$ gene, or the part sequence of $\mathrm{O}$ gene, Table S1, Supporting Information online) were constructed by Xiamen Zeesan Biotech (China). Briefly, the recombinant plasmids encoding a RNA sequence and the phage coat protein were transformed in Escherichia coli and then expressed by adding the expression inducer. After cell lysis, the armored RNA particles were separated and quantified by reverse transcription-polymerase chain reaction (RT-PCR).

\subsection{Primers design}

Based on the published sequence of SARS-CoV-2 (GenBank accession number MN908947.3), the primer sets targeted to the N, E, and $\mathrm{O}$ genes of SARS-CoV-2 (termed as N, E, and $\mathrm{O}$ primer set, respectively) were designed using PrimerExplorer V4 software (http://primerexplorer.jp/e/). Each primer set consists of the forward outer primer (F3), the backward outer primer (B3), the forward inner primer (FIP), the backward inner primer (BIP), and the loop primers (LF and/or LB) (Figure 2(a), Table S2). The melting temperature $\left(T_{\mathrm{m}}\right)$, Gibbs free energy $(\Delta G)$, and $\mathrm{G} / \mathrm{C}$ rate of the primer sets are summarized in Table S3. They were further analyzed with RNAfold web server (http://rna.tbi.univie.ac.at/cgi-bin/ RNAWebSuite/RNAfold.cgi) to ensure the absence of hairpins and self-complementarity.

\section{$2.4 \quad$ RT-LAMP in tube}

The performance of the designed primer sets for RT-LAMP detection of target sequences was tested in tube. SARS-CoV2 armored RNA particles loaded with RNA sequence of $\mathrm{N}$, $\mathrm{E}$, or $\mathrm{O}$ gene were suspended in $4 \mu \mathrm{L}$ Tris buffer at a concentration of $10^{6}$ copies/ $\mu \mathrm{L}$ and mixed with $4 \mu \mathrm{L}$ lysis buffer. After $10 \mathrm{~min}$ incubation at room temperature, the lysed sample $(8 \mu \mathrm{L})$ was mixed with $2.5 \mu \mathrm{L}$ corresponding primer set (N or O: $16 \mu \mathrm{M}$ FIP, $16 \mu \mathrm{M}$ BIP, $8 \mu \mathrm{M}$ LB, $2 \mu \mathrm{M}$ F3, and $2 \mu \mathrm{M}$ B3; E: $16 \mu \mathrm{M}$ FIP, $16 \mu \mathrm{M}$ BIP, $8 \mu \mathrm{M}$ LF, $8 \mu \mathrm{M}$ LB, $2 \mu \mathrm{M} \mathrm{F} 3$, and $2 \mu \mathrm{M} \mathrm{B} 3), 12.5 \mu \mathrm{L} 2 \times$ reaction buffer, $1 \mu \mathrm{L}$ enzyme mixture, and $1 \mu \mathrm{L}$ calcein fluorescence indicator in a micro centrifuge tube, resulting in a total reaction volume of $25 \mu \mathrm{L}$. The primer and PC RNA in the commercial kit were used as positive control (PC). Tris buffer was used as negative control (NC). The RT-LAMP reaction was performed by heating the micro centrifuge tube in a metal bath heater at $65^{\circ} \mathrm{C}$ for $60 \mathrm{~min}$, and stopped by a water bath at $95^{\circ} \mathrm{C}$ for $2 \mathrm{~min}$. The colorimetric results were directly captured under
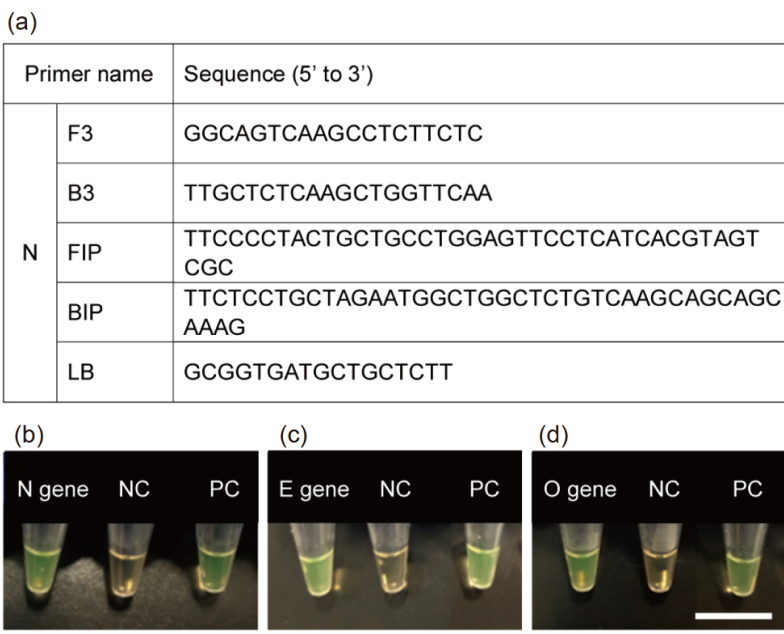

Figure 2 RT-LAMP for detecting N, E and O genes of SARS-CoV-2. (a) The primer set for N gene. Photographs of RT-LAMP in tubes using $10^{6} / \mu \mathrm{L}$ SARS-CoV-2 armored RNA particles with $\mathrm{N}$ gene and $\mathrm{N}$ gene primer set (b), E gene and $\mathrm{E}$ gene primer set (c) and $\mathrm{O}$ gene and $\mathrm{E}$ gene primer set (d). All samples generated signals appearing as green after amplification. Tris buffer was used as NC. The primers and PC-RNA in the commercial kit were used as PC. Scale bar, $1 \mathrm{~cm}$ (color online).

bright field using a camera.

For the sensitivity test, SARS-CoV-2 armored RNA particles loaded with RNA sequence of $\mathrm{N}$ gene were prepared at different concentrations of $1-10^{4}$ copies/ $\mu \mathrm{L}$ in Tris buffer and subjected to RT-LAMP following the above procedures using $\mathrm{N}$ primer set. After the completion of RT-LAMP, the fluorescence signal arising from calcein was detected under the excitation at $254 \mathrm{~nm}$ by a hand-held UV-flashlight and the colorimetric signal was directly detected under bright field.

\subsection{Design and fabrication of the centrifugal micro- fluidic disc}

The centrifugal microfluidic disc consisting of 21 identical reaction units is designed for multiplex viral nucleic acid testing. The disc is assembled from three layers: Channel layer I with embedded reaction units, Channel layer II with sample and reagent injection holes, and the sealing layer from bottom to top. Channel layer I is a $2 \mathrm{~mm}$ thick poly (methyl methacrylate) (PMMA) layer with 21 identical reaction units. Each unit contains 3 chambers: Chamber I for loading reagents, Chamber II for loading sample, and Chamber III for nucleic acid release and RT-LAMP reaction the disc center to the edge. All the chambers have a uniform depth of $1 \mathrm{~mm}$. The diameter of Chamber I and Chamber II is $5 \mathrm{~mm}$. The length and width of Chamber III are 10.5 and $5 \mathrm{~mm}$. The connection channels between chambers are $1 \mathrm{~mm}$ wide and $0.5 \mathrm{~mm}$ deep. Channel layer II is a $2 \mathrm{~mm}$ thick PMMA layer with 21 sample injection holes $(3 \mathrm{~mm}$ in diameter) and 21 reagent injection holes (5 $\mathrm{mm}$ in diameter). 
The sealing layer is a poly(dimethylsiloxane) (PDMS) layer for preventing the aerosol contamination during the entire testing procedures. All the PMMA layers were fabricated using a computer numerical control (CNC) machine. The channel layer and sealing layer were bonded at $80^{\circ} \mathrm{C}$ and $2000 \mathrm{~N}$ force conditions using vacuum hot press bonding machine (WH-2000A, Wenhao, China).

\subsection{Instrumentation}

The automated instrument for sample-to-answer detection of viral nucleic acids is composed of multiple functional modules for automated reagent injection, centrifugationbased flow actuation, temperature control, and fluorescence imaging. The overall size of the customized instrument is $360 \mathrm{~mm}$ (depth) $\times 616 \mathrm{~mm}$ (width) $\times 526 \mathrm{~mm}$ (height). The injection module is equipped with syringe pumps (LEAD FLUID, China) for loading different reagents and 4 reservoirs for washing buffer $(50 \mathrm{~mL}$ in volume, DOW CORNING, USA). The precision of automated reagent injection is ensured by a linear module (Dizi, China) and a linear module controller (Vince, China). In the flow actuation module, the high-speed rotation of the microfluidic disc is precisely controlled by a servo motor (Fuji Electric, Japan) and a servo motor controller (Fuji, Japan). The temperature control module consists of a proportional-integral-derivative (PID) temperature controller (Yexian, China), 4 thermo electric coolers (Shenzhen TECooler, China), and 4 water coolers (Beijing Jizhi, China). The fluorescence imaging module is equipped with a light-emitting diode (LED) light source ( $3 \mathrm{~W}$ power, Mshot, China), a fluorescence filter $\left(E_{\mathrm{x}} /\right.$ $E_{\mathrm{m}}: 375 / 460 \mathrm{~nm}$, Chroma, USA), an objective lens $(0.08$ numerical aperture, $6.2 \mathrm{~mm}$ working distance, OLYMPUS, Japan), an electric autofocus system (20 mm focusing range, HIWIN, China), and a CMOS camera (84\% quantum efficiency, TUCSEN, China). The fluorescence signal recorded by the imaging module was automatically processed by a build-in program. These functional modules are controlled by an industrial personal computer (ZhanMei, China) for instrument automation. The instrument is also integrated with a 13-inch display (Weichensi, China) to provide a touchscreen user interface (LabVIEW, 2019, USA) for monitoring the workflow of sample-to-answer nucleic acid testing and a real-time view of fluorescence signal at the reaction unit.

\subsection{Nucleic acid detection using the automated in- strument}

For the sample-to-answer detection of viral nucleic acids, $4 \mu \mathrm{L}$ sample fluid was injected into the Chamber II through the sample injection hole and sealed by silica gel. Meanwhile, the lysis buffer, RT-LAMP reagents (composed of $\mathrm{N}$ primer set, reaction buffer, enzyme mix, and calcein fluorescence indicator), and mineral oil were preloaded into different syringes controlled by the reagent injection module. The sample-contained microfluidic disc was then mounted in the instrument, followed by an automated workflow for RTLAMP detection of viral nucleic acids: (1) controlled the syringe to puncture through the PDMS sealing layer and injected $4 \mu \mathrm{L}$ lysis buffer into Chamber I; (2) rotated the microfluidic disc at $3000 \mathrm{r} / \mathrm{min}$ for $3 \mathrm{~s}$ to centrifuge both the sample and lysis buffer into Chamber III and incubated at room temperature for $10 \mathrm{~min}$ to release the nucleic acids; (3) injected $17 \mu \mathrm{L}$ RT-LAMP reagent into Chamber I; (4) rotated the microfluidic disc at $3000 \mathrm{r} / \mathrm{min}$ for $20 \mathrm{~s}$ to mix the lysed sample with the RT-LAMP reagent in Chamber III; (5) injected $20 \mu \mathrm{L}$ mineral oil into Chamber I and rotated the microfluidic disc at $3000 \mathrm{r} / \mathrm{min}$ for $3 \mathrm{~s}$. This procedure was performed by twice to completely fill Chamber III with mineral oil for tight sealing; (6) heated the microfluidic disc at $65{ }^{\circ} \mathrm{C}$ for $60 \mathrm{~min}$ to carry out RT-LAMP; (7) captured the fluorescence signal of Chamber III and automatically calculated the fluorescence intensity.

For the specificity test, two types of plasmids containing $\mathrm{N}$ genes of SARS-CoV or SARS-CoV-2 $\left(10^{2}\right.$ copies/ $\left.\mu \mathrm{L}\right)$ were suspended in DNase-free water to serve as the samples. DNase-free water was used as the NC. For the sensitivity test, SARS-CoV-2 armored RNA particles loading with $\mathrm{N}$ gene were suspended in Tris buffer at different concentrations $\left(0.5,1,10,10^{2}\right.$, and $10^{3}$ copies $\left./ \mu \mathrm{L}\right)$ to serve as the samples. Tris buffer was used as NC. To explore feasibility in virus detection at clinical setting, oropharyngeal swab samples from healthy volunteer and spiked with SARS-CoV-2 armored RNA particles containing $\mathrm{N}$ gene $\left(0.5\right.$ and $10^{3}$ copies $/ \mu \mathrm{L}$ ) were used as samples. Tris buffer was used as NC.

Theoretically, a given sample that contains at least one copy of the target RNA will be detected as positive by LAMP. In our case, the probability $p$ to successfully detect target RNA from $4 \mu \mathrm{L}$ sample containing 2 copies on average was estimated to be $87 \%$ using the Poisson distribution

$p=1-\left.\frac{\lambda^{k} e^{-\lambda}}{k !}\right|_{k=0}=87 \%$

where $\lambda=2$ is the average number of target RNA per $4 \mu \mathrm{L}$ sample and $k=0$ represents the absence of target RNA within the sample.

\subsection{Computational fluid dynamic simulation}

To investigate the impact-induced mixing between sample and reagent solutions within the reaction unit, the computational fluid dynamic (CFD) simulation was performed using a finite-volume solver (Fluent 6.4, ANSYS Inc., USA). The motion of liquid and air phases within the reaction unit was obtained by using the volume of fluid (VOF) model, in which 
continuity and momentum equations depending on the volume fraction of each phase were solved:

$$
\begin{aligned}
& \frac{\partial \rho}{\partial t}+\nabla \cdot(\rho \mathbf{u})=0 \\
& \frac{\partial(\rho \mathbf{u})}{\partial t}+\nabla \cdot(\rho \mathbf{u u})=-\nabla p+\nabla \cdot(\eta \nabla \mathbf{u})+\rho \mathbf{g}+\mathbf{F} \\
& \mathbf{u}=\frac{\sum \alpha_{i} \rho_{i} \mathbf{u}_{i}}{\Sigma \alpha_{i} \rho_{i}}, \rho=\Sigma \alpha_{i} \rho_{i}
\end{aligned}
$$

where $\alpha_{i}, \rho_{i}$, and $\mathbf{u}_{i}$ are the volume fraction, density, and velocity vector of $i$-th phase, respectively, $p$ is the pressure, $\eta$ is the dynamic viscosity of fluid, $\mathbf{g}$ is the vector of the centrifugal acceleration, and $\mathbf{F}$ is the surface tension force per unit volume. To solve the equations, least square cell based gradient was used for spatial discretization, QUICK scheme was used for momentum discretization, and PRESTO was used for pressure discretization. Time step was set to be $1 \mu \mathrm{s}$.

\section{Results and discussion}

\subsection{Centrifugal microfluidic system for viral nucleic acid testing}

The fully automated centrifugal microfluidic system for sample-to-answer detection of viral nucleic acids is comprised of a customized instrument, an integrated microfluidic disc, and a reagent set for the release of nucleic acids and RTLAMP detection (Figure 1, Figure S1, Supporting Information online). The instrument consists of multiple functional modules for automated reagent injection, centrifugationbased flow actuation, temperature control, fluorescence imaging, and data processing, allowing for automatically streamlining the entire procedures of viral nucleic acid testing. The microfluidic disc with 21 independent reaction units was loaded with virus samples and then mounted onto the automated instrument. Virus lysis buffer, RT-LAMP reagents, and mineral oil were sequentially injected into the microfluidic disc for on-chip release of viral nucleic acids, amplification of target RNA, and sealing of reaction unit. After each injection, the microfluidic disc was under program-controlled high-speed rotation for actuating and mixing different fluids. The on-chip release of viral nucleic acids was performed at room temperature for $10 \mathrm{~min}$, and RTLAMP reaction was at $65^{\circ} \mathrm{C}$ up to 60 min using PID temperature controller. Fluorescence signal indicating the presence of target RNA was recorded by the imaging module and processed by the built-in program. This automated centrifugal microfluidic system enables the sample-toanswer detection of viral nucleic acids in $70 \mathrm{~min}$ with minimal operation.

\subsection{RT-LAMP in tube}

We designed three primer sets targeted to N, E and O genes of SARS-CoV-2 (Figure 2, Table S2) and tested their performance for RT-LAMP detection of target sequences in tube. The primers and PC RNA in the commercial kit were used as PC. Tris buffer was used as NC. $10^{6} / \mu \mathrm{L}$ SARS-CoV2 armored RNA particles loaded with RNA sequences of N, $\mathrm{E}$ or $\mathrm{O}$ gene were first treated with the lysis buffer to release nucleic acids at room temperature for $10 \mathrm{~min}$, and then subjected to RT-LAMP at $65{ }^{\circ} \mathrm{C}$ for $60 \mathrm{~min}$. As shown in Figure 2, all samples generated signals appearing as green after amplification, indicating that the $\mathrm{N}, \mathrm{E}$ and $\mathrm{O}$ primer sets can be used for RT-LAMP in tube.

As SARS-CoV-2 shows a higher transcript level of $\mathrm{N}$ gene than that of $\mathrm{O}$ gene, the detection of $\mathrm{N}$ gene may provide better diagnostic performance [39]. To assess the sensitivity of RT-LAMP for detecting N gene of SARS-CoV-2, serial dilution test was performed using armored RNA particles with $\mathrm{N}$ gene. After lysis treatment and RT-LAMP, the bright green fluorescence for armored RNA particles from 1 to $10^{4}$ copies $/ \mu \mathrm{L}$ was observed in tubes using the $\mathrm{N}$ primer set, indicating the high sensitivity of RT-LAMP (Figure 3).

\subsection{Centrifugal microfluidic disc}

The centrifugal microfluidic disc has 21 identical reaction units enabling the multiplex analysis of viral nucleic acids with sample-in-answer-out capability (Figure 4(a)). Each reaction unit consists of 3 chambers, including a chamber for loading reagents (Chamber I), a chamber for loading samples (Chamber II), and a reaction chamber (Chamber III) from the disc center to the edge. After loading the samples by pipette into Chamber II, the microfluidic disc was tightly sealed by the silica gel (Figure 4(b)). Virus lysis buffer, RT-LAMP reagents, and mineral oil were sequentially injected into

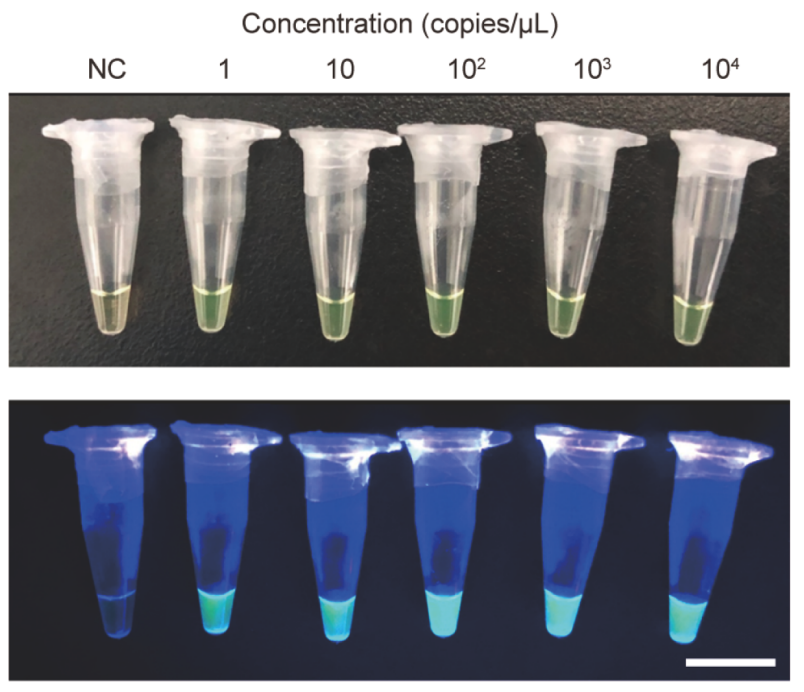

Figure 3 Colorimetric and fluorescent detection of $\mathrm{N}$ gene in armored RNA particles at different concentrations of $1-10^{4}$ copies $/ \mu \mathrm{L}$ using the $\mathrm{N}$ primer set. Tris buffer was used as NC. Scale bar, $1 \mathrm{~cm}$ (color online). 
(a)

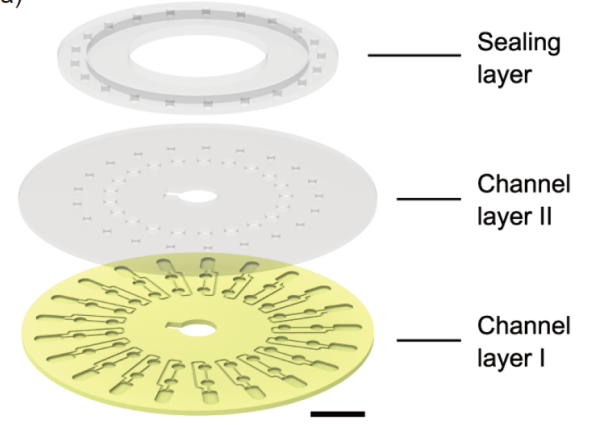

(b)

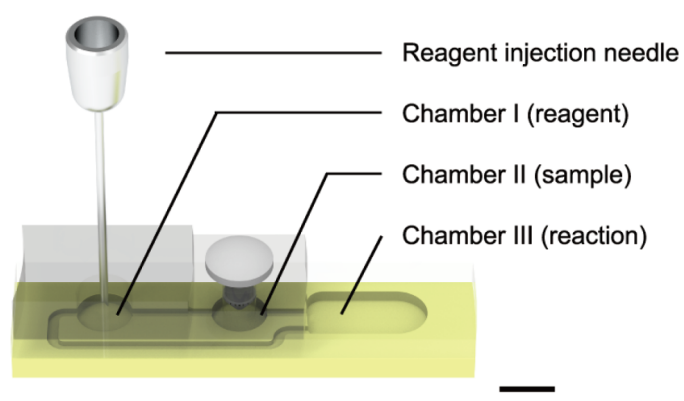

(c)

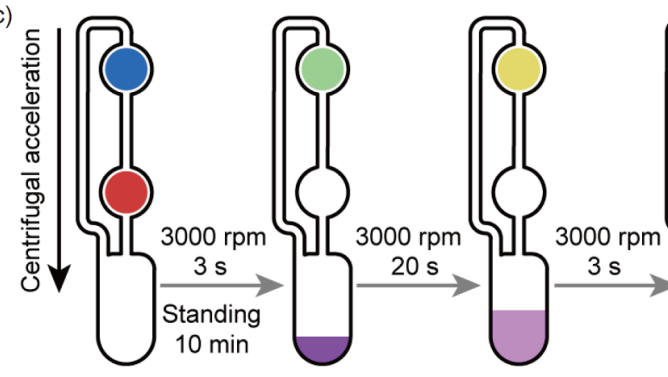

Figure 4 Centrifugal microfluidic disc for viral nucleic acid testing. (a) Layer-by-layer structure of the microfluidic disc. From bottom to top: Channel layer I, Channel layer II, and the sealing layer. Scale bar, $2 \mathrm{~cm}$. (b) The structure of reaction unit consisting of 3 chambers. From disc center to edge: Chamber I for reagent injection, Chamber II for sample injection, and Chamber III for nucleic acid release and RT-LAMP reaction. Scale bar, $0.5 \mathrm{~cm}$. (c) Step-by-step illustration of viral nucleic acid release, amplification, and detection within the integrated microfluidic disc (color online).

Chamber I. The release of viral nucleic acids and amplification of target RNA were performed in Chamber III. The fluorescence signal indicating the presence of target RNA can be directly observed in Chamber III as the entire microfluidic disc was made from transparent materials (PMMA and PDMS). The sealing of reaction unit was tested by heating the microfluidic chip at $65^{\circ} \mathrm{C}$ for $60 \mathrm{~min}$ (to mimic LAMP amplification) (Figure S2). No leakage or liquid evaporation was observed as Rhodamine B liquid segment inside the reaction unit remained unchanged. The complete seal of reaction unit ensured that the amplicons will not become aerosol to contaminate the next LAMP.

To enable the rapid, on-demand mixing of samples with reagents in the Chamber III, two adjacent chambers were bridged by a microchannel with $1000 \mu \mathrm{m}$ in width $(w)$ and $500 \mu \mathrm{m}$ in depth $(h)$. Under stagnant conditions, a convex liquid front was formed at the junction of the microchannel and the chamber due to the sudden expansion configuration, resulting in a pressure barrier $(\Delta p)$ directing toward the liquid phase to stop the flow [40]:

$$
\begin{aligned}
\Delta p & =\frac{2 \gamma}{w}\left[-\frac{w}{h} \cos \theta_{c}-\cos \left(\min \left\{\theta_{c}+\beta, 180^{\circ}\right\}\right)\right] \\
\omega & =\sqrt{\frac{\Delta p}{\rho r_{m} \Delta r}}
\end{aligned}
$$

where $\gamma=7.2 \times 10^{-2} \mathrm{~N} / \mathrm{m}$ is the liquid-air surface tension, $\theta_{c}=71^{\circ}$ is the contact angle, $\beta=90^{\circ}$ is the wedge angle of the sudden expansion, $\rho=10^{3} \mathrm{~kg} / \mathrm{m}^{3}$ is the fluid density, $r_{m}$ is the average distance between liquid front, and $\Delta r$ is the length of the liquid body. Given an $r_{m}$ of $32 \mathrm{~mm}$ and a $\Delta r$ of $2.3 \mathrm{~mm}$, the minimum rotation rate required to overcome the pressure barrier was estimated to be $229 \mathrm{r} / \mathrm{min}$. Therefore, the setting rotation rate of $3000 \mathrm{r} / \mathrm{min}$ was high enough to actuate the fluid into Chamber III (Figure S3). CFD simulation revealed an impact-induced mixing between sample and reagent solutions at $3000 \mathrm{r} / \mathrm{min}$, which significantly reduced the characteristic diffusion length to $\sim 0.1 \mathrm{~mm}$ within $55 \mathrm{~ms}$ (Figure 5). The minimal diffusion time for complete mixing was estimated to be $17 \mathrm{~s}$ using the following equation:

$t=\frac{L^{2}}{6 D}$

where $L=0.1 \mathrm{~mm}$ is the characteristic diffusion length and $D=10^{-10} \mathrm{~m}^{2} / \mathrm{s}$ is the diffusion coefficient estimated for Bst enzyme $(66.5 \mathrm{kDa})$.

\subsection{Automated instrumentation}

For sample-in-answer-out detection of viral nucleic acids, an automated, stand-alone instrument was designed (Figure 6 (a)). The instrument is composed of (1) syringe pumps equipped with injection needles for loading reagents; (2) a motor for rotating the microfluidic disc at high speed; (3) a temperature controlling system for carrying out RT-LAMP reaction; (4) an imaging system for real-time quantifying the fluorescence signal; and (5) an industrial personal computer for controlling the modules to streamline the entire procedures. The operator only needs to pipette the samples into the microfluidic disc and mount it into the instrument for rapid 


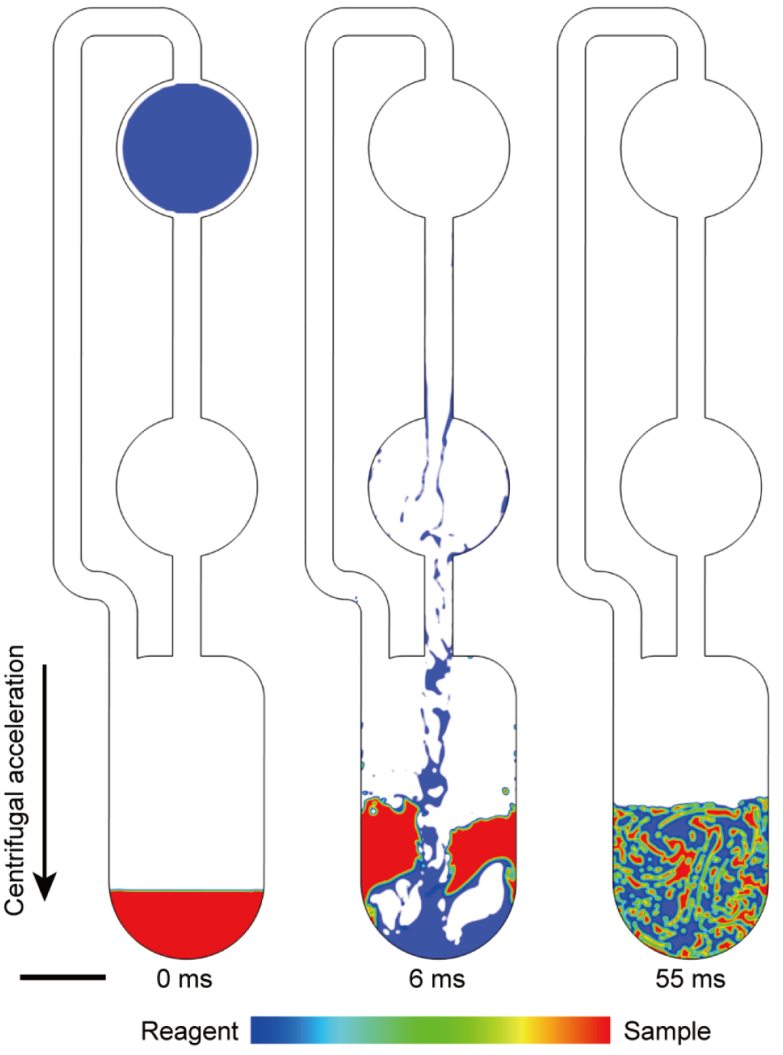

Figure 5 CFD simulation of an impact-induced mixing between sample and reagent solutions at $3000 \mathrm{r} / \mathrm{min}$. Scale bar, $3 \mathrm{~mm}$ (color online).

detection of viral nucleic acids, without the requirement of skilled personnel and clinical diagnostic laboratory.

To characterize the performance of customized instrument, its accuracy for reagent injection was first tested (Figure 6 (b)). We set the injection volumes to be $4 \mu \mathrm{L}$ (for nucleic acid release reagents) and $17 \mu \mathrm{L}$ (for LAMP reagents), and measured the actual volumes after automated injection. A standard deviation of $<2 \%$ was obtained, showing the good accuracy and reproducibility of the injection system. We next assessed the rotation stability of instrument (Figure 6(c)). By setting the rotation rate at $3000 \mathrm{r} / \mathrm{min}$ for $3 \mathrm{~s}$, we observed a sudden increase in measured rotation rate from 0 to $3000 \mathrm{r} / \mathrm{min}$, followed by a plateau at $3000 \mathrm{r} / \mathrm{min}$ for $3 \mathrm{~s}$. In addition, a temperature of $65^{\circ} \mathrm{C}$ (for RT-LAMP reaction) could be maintained more than 90 min using the temperature controlling system (Figure 6(d)). The imaging system for collecting signals showed an intra-unit variation of $<2 \%$ and an inter-unit variation of $<3 \%$ (Figure 6(e)).

\subsection{Automated detection of nucleic acids}

To demonstrate the automated detection of viral nucleic acids by the centrifugal microfluidic system, we loaded different concentrations of SARS-CoV-2 armored RNA particles with $\mathrm{N}$ gene $\left(0.5,1,10,10^{2}, 10^{3}\right.$ copies $\left./ \mu \mathrm{L}\right)$ into five reaction units of the microfluidic disc, and used the instrument to carry out on-chip release of viral nuclei acids, RT-LAMP, and realtime fluorescence signal detection. Tris buffer was used as NC. The amplification curve of armored RNA particles at $10^{3}$ copies $/ \mu \mathrm{L}$ showed a sharp increase at $\sim 35 \mathrm{~min}$ and reached a maximum fluorescent intensity at $45 \mathrm{~min}$ after amplification (Figure 7(a)). All samples with different RNA concentrations from 0.5 to $10^{3}$ copies $/ \mu \mathrm{L}$ could be amplified and detected within $55 \mathrm{~min}$. The limit of detection of this automated centrifugal microfluidic system was 2 copies per reaction for detecting SARS-CoV-2 armored RNA particles. The entire assay sample-to-answer time was less than $70 \mathrm{~min}$.

To test the specificity of the microfluidic assay, plasmids containing $\mathrm{N}$ gene of SARS-CoV or SARS-CoV-2 were used. Based on the analysis using the Basic Local Alignment Search Tool (BLAST), the amplification sequences of $\mathrm{N}$ gene of SARS-CoV and SARS-CoV-2 had a similarity of 87\% (Figure S4). As shown in Figure 7(b), SARS-CoV-2 plasmids $\left(10^{2}\right.$ copies $\left./ \mu \mathrm{L}\right)$ could be detected by the automated microfluidic system, whereas SARS-CoV plasmids $\left(10^{2}\right.$ copies $/ \mu \mathrm{L}$ ) and negative control (NC, water) could not be amplified (Figure 7(b)). This is attributed to the use of LAMP primers that specifically recognize six regions of target sequences, allowing for high specificity detection of SARS-CoV-2 using the automated microfluidic system.

We next demonstrated the practical applications of automated microfluidic system for viral detection using oropharyngeal swab samples from healthy volunteer and spiked with SARS-CoV-2 armored RNA particles $\left(0.5,10^{3}\right.$ copies $/ \mu \mathrm{L}$ ) (Figure $8(\mathrm{a})$ ). No fluorescence signals were detected for the swab sample without armored RNA particles as well as for NC (tris buffer) after microfluidic sample treatment and RT-LAMP (Figure 8(b)). In contrast, the swab sample spiked with even 0.5 copies $/ \mu \mathrm{L}$ armored RNA particles showed a positive signal. These results collectively demonstrate that the automated microfluidic system with good performance can be adapted to detect viral nucleic acids in a sample-to-answer manner.

\section{Conclusions}

In this work, we developed a fully automated centrifugal microfluidic system for sample-to-answer detection of viral nucleic acids in 70 min with minimal operation. The microfluidic system was comprised of a customized instrument, an integrated microfluidic disc, and a reagent set for the release of nucleic acids and RT-LAMP detection. The limit of detection for SARS-CoV-2 armored RNA particles was 2 copies per reaction, and the assay did not cross-react with SARS-CoV. The oropharyngeal swab samples spiked with SARS-CoV-2 armored RNA particles down to 0.5 copies $/ \mu \mathrm{L}$ 

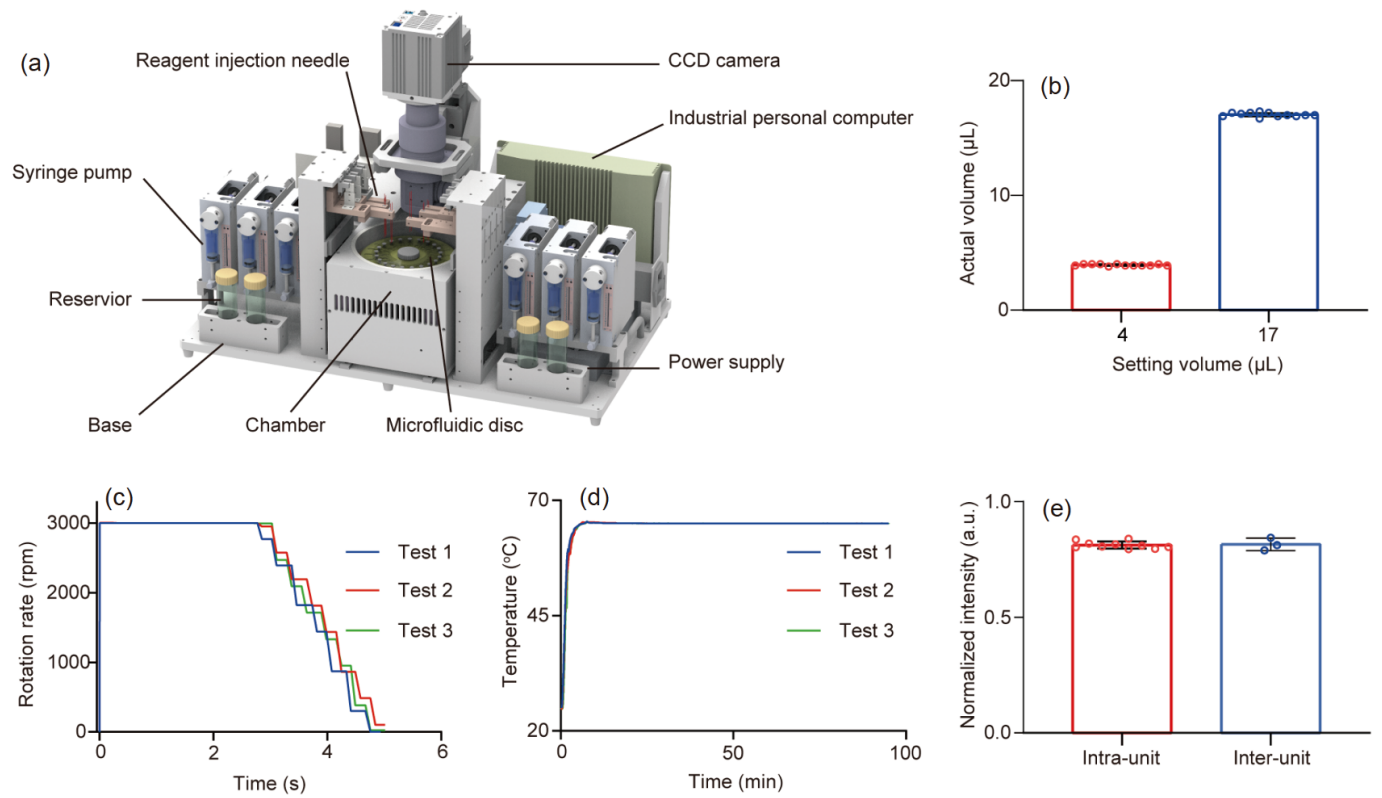

Figure 6 Characterization of customized, stand-alone instrument. (a) Components of the instrument; (b) accuracy test for reagent injection; (c) rotation stability test; (d) temperature control test; (e) signal collection test (color online).
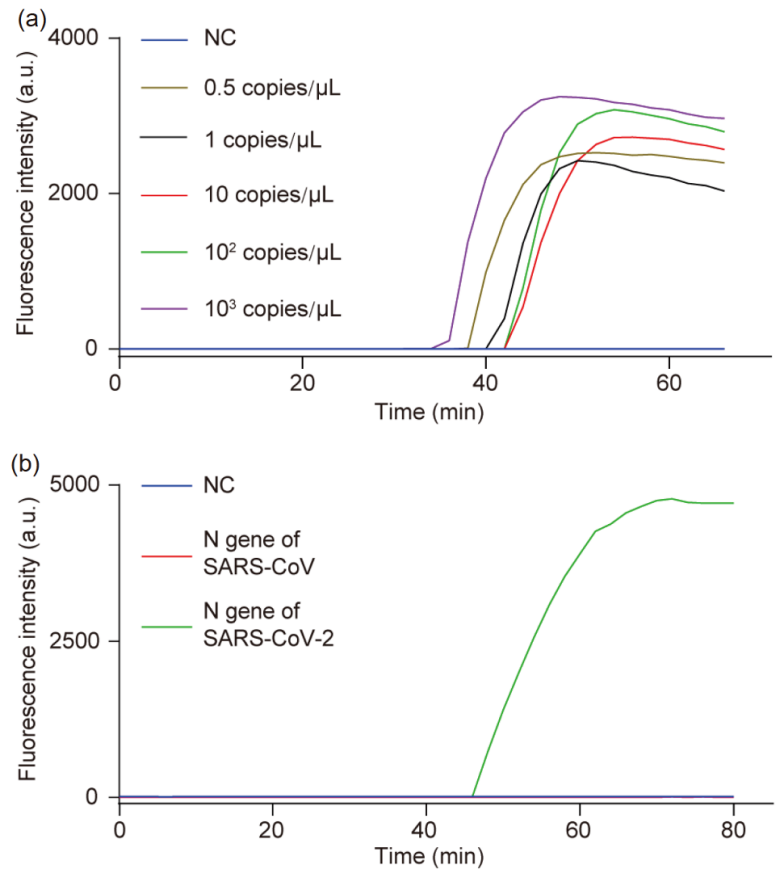

Figure 7 Highly sensitive detection of viral nucleic acids using the automated instrument. (a) The amplification curve of SARS-CoV-2 armored RNA particles with $N$ gene $\left(0.5,1,10,10^{2}, 10^{3}\right.$ copies $\left./ \mu \mathrm{L}\right)$ inside the microfluidic disc. Tris buffer was used as NC. (b) The amplification curve of plasmids containing $\mathrm{N}$ gene of SARS-CoV or SARS-CoV-2 $\left(10^{2}\right.$ $\operatorname{copies} / \mu \mathrm{L})$. Water was used as NC (color online).

could be detected. To make our system more compatible to NAAT outside the diagnostic laboratory, technical improvements are required to further reduce the instrument size, including minimizing the diameter of the microfluidic (a)

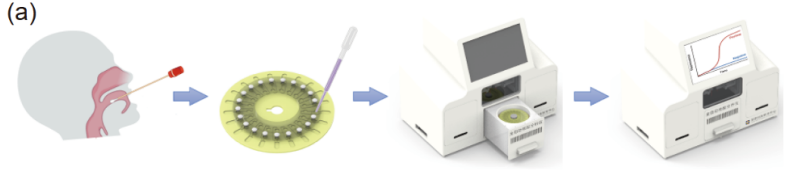

(b)

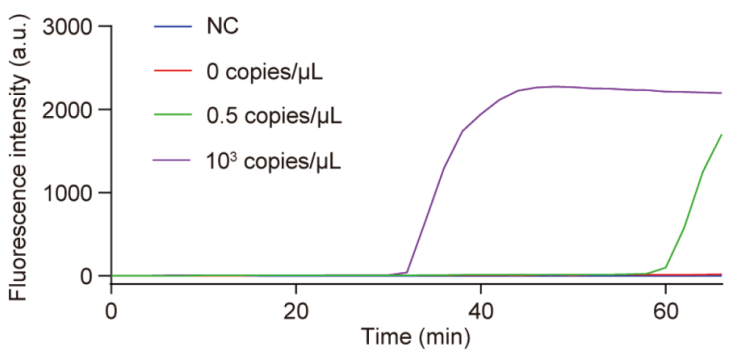

Figure 8 Automated microfluidic system for viral detection using oropharyngeal swab samples. (a) Procedures of sample collection and viral nucleic acid testing. (b) The amplification curve of oropharyngeal swab samples from healthy volunteer and spiked with SARS-CoV-2 armored RNA particles $\left(0.5,10^{3}\right.$ copies $/ \mu \mathrm{L}$ ). Tris buffer was used as NC (color online).

disc by using higher rotation speed, simplifying flow control modulus by preloading lyophilized reagents in the microfluidic device, and optimizing the arrangement of optical path and using photomultiplier tube instead of CMOS camera for fluorescence detection. We envisioned that this microfluidic system free of aerosol contamination may facilitate viral nucleic acid detection outside the diagnostic laboratory, promoting diagnosis efficiency of infectious diseases and preventing onward transmission.

Acknowledgements This work was supported by the National Natural Science Foundation of China (91959101, 21904028), Chinese Academy of 
Sciences (YJKYYQ20180055, YJKYYQ20190068, ZDBS-LY-SLH025), and the Strategic Priority Research Program of Chinese Academy of Sciences (XDB36000000).

Conflict of interest The authors declare no conflict of interest.

Supporting information The supporting information is available online at http://chem.scichina.com and http://link.springer.com/journal/11426. The supporting materials are published as submitted, without typesetting or editing. The responsibility for scientific accuracy and content remains entirely with the authors.

1 Organization WH. World Health Statistics 2019: Monitoring Health for the SDGs, Sustainable Development Goals. Geneva: World Health Organization, 2019

2 Fournier PE, Drancourt M, Colson P, Rolain JM, La Scola B, Raoult D. Nat Rev Microbiol, 2013, 11: 574-585

3 Wang Y, Ruan Q, Lei ZC, Lin SC, Zhu Z, Zhou L, Yang C. Anal Chem, 2018, 90: 5224-5231

4 He P, Lv F, Liu L, Wang S. Sci China Chem, 2017, 60: 1567-1574

5 Land KJ, Boeras DI, Chen XS, Ramsay AR, Peeling RW. Nat Microbiol, 2019, 4: 46-54

6 Zhang M, Li R, Wang J, Ling L. Sci China Chem, 2017, 60: 14681473

7 Dou M, Macias N, Shen F, Dien Bard J, Domínguez DC, Li XJ. EClinicalMedicine, 2019, 8: 72-77

8 Dou M, Sanchez J, Tavakoli H, Gonzalez JE, Sun J, Dien Bard J, Li XJ. Anal Chim Acta, 2019, 1065: 71-78

9 Yang B, Kong J, Fang X. Talanta, 2019, 204: 685-692

10 Yang B, Fan Y, Li Y, Yan J, Fang X, Kong J. Analyst, 2020, 145: 3814-3821

11 Zhu Z, Zhang W, Leng X, Zhang M, Guan Z, Lu J, Yang CJ. Lab Chip, 2012, 12: 3907-3913

12 Wu W, Zhang T, Han D, Fan H, Zhu G, Ding X, Wu C, You M, Qiu L, Li J, Zhang L, Lian X, Hu R, Mu Y, Zhou J, Tan W. Chem Sci, 2018, 9: $3050-3055$

13 Hu Y, Xu P, Luo J, He H, Du W. Anal Chem, 2017, 89: 745-750

14 Broughton JP, Deng X, Yu G, Fasching CL, Servellita V, Singh J, Miao X, Streithorst JA, Granados A, Sotomayor-Gonzalez A, Zorn K, Gopez A, Hsu E, Gu W, Miller S, Pan CY, Guevara H, Wadford DA, Chen JS, Chiu CY. Nat Biotechnol, 2020, 38: 870-874

15 Yu M, Chen X, Qu H, Ma L, Xu L, Lv W, Wang H, Ismagilov RF, Li M, Shen F. Anal Chem, 2019, 91: 8751-8755

16 Dou M, Sanjay ST, Dominguez DC, Zhan S, Li XJ. Chem Commun, 2017, 53: 10886-10889
17 Wang TZ, Zhang Y, Huang GL, Wang C, Xie L, Ma L, Li ZY, Luo XB, Tian H, Li Q, Li X, Lv ZY, Bao XF. Sci China Chem, 2012, 55: $508-514$

18 Wu L, Ding H, Qu X, Shi X, Yang J, Huang M, Zhang J, Zhang H, Song J, Zhu L, Song Y, Ma Y, Yang C. J Am Chem Soc, 2020, 142: 4800-4806

19 Li X, Zhang D, Zhang H, Guan Z, Song Y, Liu R, Zhu Z, Yang C. Anal Chem, 2018, 90: 2570-2577

20 Feng Q, Zhang L, Liu C, Li X, Hu G, Sun J, Jiang X. Biomicrofluidics, 2015, 9: 052604

21 Feng Q, Sun J, Jiang X. Nanoscale, 2016, 8: 12430-12443

22 Zhang H, Liu Y, Wang J, Shao C, Zhao Y. Sci China Chem, 2019, 62: 87-94

23 Chen P, Chen C, Liu Y, Du W, Feng X, Liu BF. Sens Actuat B-Chem, 2019, 283: 472-477

24 Li L, Geng Y, Xiang Y, Qiang H, Wang Y, Chang J, Zhao H, Zhang L. Anal Chim Acta, 2019, 1062: 102-109

25 Yi Q, Cai D, Xiao M, Nie M, Cui Q, Cheng J, Li C, Feng J, Urban G, Xu YC, Lan Y, Du W. Biosens Bioelectron, 2019, 135: 200-207

26 Feng Q, Liu J, Li X, Chen Q, Sun J, Shi X, Ding B, Yu H, Li Y, Jiang X. Small, 2017, 13: 1603109

27 Cai D, Xiao M, Xu P, Xu YC, Du W. Lab Chip, 2014, 14: 3917-3924

28 Wei X, Zhou W, Sanjay ST, Zhang J, Jin Q, Xu F, Dominguez DC, Li XJ. Anal Chem, 2018, 90: 9888-9896

29 Dou M, Sanjay ST, Dominguez DC, Liu P, Xu F, Li XJ. Biosens Bioelectron, 2017, 87: 865-873

30 Jiang X, Loeb JC, Manzanas C, Lednicky JA, Fan ZH. Angew Chem Int Ed, 2018, 57: 17211-17214

31 Song J, Mauk MG, Hackett BA, Cherry S, Bau HH, Liu C. Anal Chem, 2016, 88: 7289-7294

32 Ahmed MG, Abate MF, Song Y, Zhu Z, Yan F, Xu Y, Wang X, Li Q, Yang C. Angew Chem Int Ed, 2017, 56: 10681-10685

33 Song J, Liu C, Mauk MG, Peng J, Schoenfeld T, Bau HH. Anal Chem, 2018, 90: 1209-1216

34 Zhang Y, Zhang L, Sun J, Liu Y, Ma X, Cui S, Ma L, Xi JJ, Jiang X. Anal Chem, 2014, 86: 7057-7062

35 Zhang L, Zhang Y, Wang C, Feng Q, Fan F, Zhang G, Kang X, Qin X, Sun J, Li Y, Jiang X. Anal Chem, 2014, 86: 10461-10466

36 Zhang L, Tian F, Liu C, Feng Q, Ma T, Zhao Z, Li T, Jiang X, Sun J. Lab Chip, 2018, 18: 610-619

37 Zhang L, Ding B, Chen Q, Feng Q, Lin L, Sun J. TrAC Trends Anal Chem, 2017, 94: 106-116

38 Ye X, Li Y, Fang X, Kong J. ACS Sens, 2020, 5: 1132-1139

39 Kim D, Lee JY, Yang JS, Kim JW, Kim VN, Chang H. Cell, 2020, 181: 914-921.e10

40 Chen JM, Huang PC, Lin MG. Microfluid Nanofluid, 2008, 4: 427437 